\title{
RAYLEIGH SCATTERING FROM SINC E-SITE POLYSYLANE ADSORBED ON SILICON: THEORY
}

\author{
C M J WIJERS \\ Department of Appled Physacs, Twente Unuersit of \\ chnologv, \\ PO Box 217, 7500 AE Enschede. The Netherlands
}

Received 10 June 1985 , accepted for publication 19 J

1985

An ordered set of dipoles with mutual interaction is studied The solution of that particular theoretical static and dynamic case It can serve as a description . surface of slicon by means of HOMOCVD or sputter can be used to describe the optical behaviour of those $r$ and next also the photoluminescence of those molecu the close vicinity of a dielectric surtace roblem is given rigorously both for the the polysylane molecules formed at the I in a hydrogen atmosphere The theory lecules, like first the Rayleigh scattering ,

\section{Introduction}

Spear and Le Comber [1] were probd present activities in the field of hydrogen that the amount of hydrogen stored in $t$ l number of dangling bonds by about a tar process more was going on then a simple analysis, especially by means of IR spectrc e g ref [2]), started to point gradually 1 $\left(\mathrm{S}_{1} \mathrm{H}_{2}\right)_{n}$-type of chains at the surtace of $t$ production of those novel materials - hom tion [3] and RF plasma sputtering [4] - ex be established Interest in hydrogenated ar lated by the discovery of its photoluminesc ted over a wider energy interval, a-S $\mathrm{H}$ e minescence intensity (tor HOMOCVD pr. tıng commercial LED materials [4]

The larger band gap, as compared to observations, asks for calculations of the assuming vacancies [7.8] or assuming form tion latter, more relevant approach, yields sat" ying gap values, but the observed

(0039-6028/86/\$0350 (c) Elsevier Science PI lishers B V

(North-Holland Physics Publishing Divisic ) y the first to contribute to the ed amorphous silicon The tact s type of samples exceeded the or of 100 , indicated that in this arface adsorption Spectroscopic zopy and Raman techniques (see to the direction of formation of se materials For both types of geneous chemical vapour deposi.tence of $\left(\mathrm{S}_{1} \mathrm{H}_{2}\right)_{n}$ chains seems to rphous silicon was further stımuit properties [4-6] Though emitubits a tive times larger photoluluced tilms) as compared to exisidinary a-Si $H$, required by such lectronic structure, as were done tion of polysylane chams [9] The 
intensities remain unexplained As such very useful information might be gathered from surface Rayleıgh scatterıng experıments

A rigorous theoretical solution of this problem being beyond the present abilities, we will base this article upon the assumptions customary in the theory of Rayleigh scattering from surfaces and from large molecules in the gas phase Concerning the latter the classical induced-dipole model is the prevalling method and here the obvious similarity between dlkanes and polysylanes has to be emphasized From alkanes it is known that a straightforward application of this model yields unrealistic results and one needs to screen the internal interactions with a certain factor $\varepsilon_{4}$ to obtain realistic results [10,1] With respect to the influence of the surface, represented by a homogeneous halfspace characterized by a complex dielectric constant $\tilde{\varepsilon}$, we will investigate two models Firstly, d static model will be used where the polysylane molecule interacts with the surface through image interaction The molecule itselt will be presumed to reside above the surface Secondly, a dynamic model will be presented based on a mathematically regorous solution for a radiatıng dipole above a dielectric surface, using the method of Sommerfeld

\section{Image field solution}

More detaled information about the ımage field method, as applied to surface Rayleigh/Raman problems can be found in ref [12] First a separate polysylane will be considered briefly in the same way as in ref [10] The polysylane will be represented by a finite number $N$ of discrete dipoles. positioned at $\boldsymbol{r}_{t}$ and connected to their local field through

$\boldsymbol{p}_{t}=\boldsymbol{\alpha}_{t} \boldsymbol{E}_{\mathrm{loc}}\left(\boldsymbol{r}_{t}\right)$

$\boldsymbol{\alpha}_{t}$ represents as usual the poldrizability of the corresponding dipole

This detines the configuration to be used, depending on whether one assigns the dipole to the atom (site model) or to the bonding (backbone model) At short distances the dipole field can be approximated by the induction field alone hence allowing for a (quasi-) static approach Now the exphicit form of (1) becomes ( $\boldsymbol{E}_{\mathrm{c},}$ represents the incoming field)

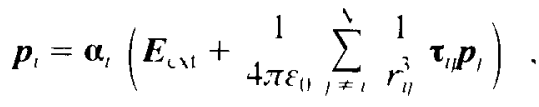

$$
\begin{aligned}
& r_{i l}=\left|\boldsymbol{r}_{t}-\boldsymbol{r}_{j}\right|, \quad \boldsymbol{\tau}_{l j}=3 \hat{\boldsymbol{r}}_{t j} \hat{\boldsymbol{r}}_{i j}-\mathbf{1}
\end{aligned}
$$

Taking into account all dipoles, (2) transtorms into a system of linear equations from which one derives, after introducing the abbreviations $\alpha_{01}=\varepsilon_{11} a^{3}$, $\mathbf{C}_{t}=\left(4 \tau \alpha_{11}\right) \boldsymbol{\alpha}_{t}^{-1}$ and $\boldsymbol{\pi}_{t}=\boldsymbol{p}_{t} / \alpha_{1}\left|\boldsymbol{E}_{\mathrm{cxt}}\right|$, the dimensionless system of interaction equations 
$\mathbf{A} \boldsymbol{\pi}=4 \pi \hat{\boldsymbol{e}}$

$\pi$ is a composite vector containing all sef rate $\pi_{i}, a$ is the Bohr radius Equivalently $\hat{\boldsymbol{e}}$ contains $N$ times the unit ve or controlling $\boldsymbol{E}_{\mathrm{c} \backslash \mathrm{t}}$ The matrix A has an $(N \times N)$ dyadic structure with expli t form

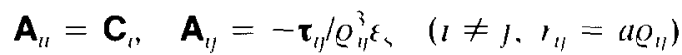

The screenng factor $\varepsilon_{4}$, being almost exactly 10 tor alkanes [11]. 1s necessary to obtain realistic results, as emphasızed in ef [10] Placing next a surface close to the polysylane molecule causes a me ification of eq (3) For a single dipole $\boldsymbol{p}$ at a height $z$ above a dielectric sur ce characterized by a dielectric constant $\tilde{\varepsilon}$, a solution fulfillıng the boundar conditions

$\boldsymbol{E}_{\|}^{+}=\boldsymbol{E}_{\|}^{-}, \quad \boldsymbol{E}_{\perp}^{+}=\tilde{\varepsilon} \boldsymbol{E}_{\perp}^{-}$

is obtained through adding an image dipole ' at $-z$ as in ref [12]

$\boldsymbol{p}^{\prime}=\boldsymbol{\eta} \boldsymbol{p}, \quad \boldsymbol{\eta}=\overline{\bar{\varepsilon}}-1\left\|\begin{array}{rrr}-1 & 0 & 0 \\ 0 & -1 & 0 \\ 0 & 0 & 1\end{array}\right\|$

where the z-axis is supposed to be perpend ilar to the surtace Solving now for the entire polysylane molecule, we req re every dipole in the chan to produce an image dipole according to (6) This procedure guarantees the obeying of the boundary conditions, throug out the procedure

Now $\boldsymbol{E}_{\mathrm{c} x t}$ is the sum of the incoming anc reflected beam So we arrive at the equivalent expression of eq (1)

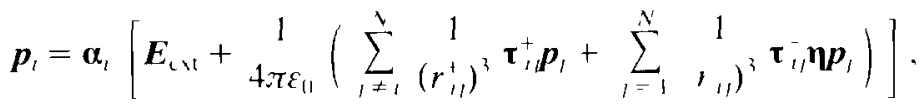

$\boldsymbol{r}_{11}^{+}=\boldsymbol{r}_{1}^{+}-\boldsymbol{r}_{1}^{+}, \quad \boldsymbol{r}_{11}=\boldsymbol{r}_{1}^{+}-\boldsymbol{r}_{1}^{-}$

Position vectors with upper index - have $z$ mponent reversed as compared to their + counterparts The dyadics tor the teraction matrix now become

$\mathbf{A}_{u l}^{\prime}=\mathbf{C}_{l}-\left(\boldsymbol{\tau}_{t \imath}^{-} \boldsymbol{\eta}\right) / \varepsilon_{\mathrm{r}}\left(Q_{11}^{-}\right)^{3}$.

$\mathbf{A}_{l \prime}^{\prime}=-\left[\boldsymbol{\tau}_{l l}^{+} / \varepsilon_{1}\left(\omega_{l l}^{+}\right)^{3}+\left(\boldsymbol{\tau}_{l l}^{-} \boldsymbol{\eta}\right) / \varepsilon_{\mathrm{r}}\left(\varrho_{l l}^{-}\right)^{3}\right]$

$\varepsilon$, has the equivalent meaning as in (4) Di the diagonal elements reduce, giving rise i remedy has to be sought in screening of th

to the influence of the surface, 1 (physisorption) to 10 (chemisorption) or $n$ unstable solutions [10.11] The image fields by $\varepsilon_{1}$, varying from ire generally as a function of z 


\section{Dynamic solution: Sommerfeld's method}

As far as the classical dynamic treatment of a single dipole above a dielectric surface is concerned, refs $[13,14]$ build a good introduction For the dynamic solution one needs Sommerfeld's method [15] with boundary cond1tions

$\boldsymbol{E}_{\|}^{+}=\boldsymbol{E}_{\|}^{-}, \quad \boldsymbol{H}_{\|}^{+}=\boldsymbol{H}_{\|}^{-}$

Using the expansion of the spherical wave in Bessel functions the result follows in a way closely resembling (7)

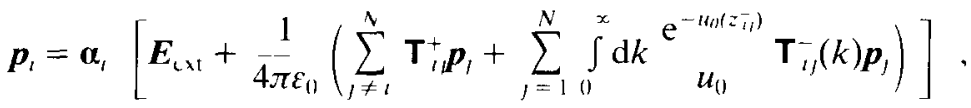

$$
\begin{aligned}
& u_{0}=\left(k^{2}-k_{0}^{2}\right)^{1 / 2}
\end{aligned}
$$

Besides previous definıtıons, we assume here an incomıng wave of frequency $\omega$, but in (10) the frequency-dependent exponential is divided out, leaving only (complex) amplitudes $k_{0}$ represents the in vacuo wavenumber It remains to define the two dyadic tensors

The first one $\mathbf{T}^{+}$governs the direct interactions between the dipoles representıng the polysylane molecule

$$
\begin{aligned}
& \mathbf{T}_{l \jmath}^{+}=g_{1}\left(r_{\imath \jmath}^{+}\right) \mathbf{1}+g_{2}\left(r_{1,}^{+}\right) \hat{\boldsymbol{r}}_{\imath,}^{+} \hat{\boldsymbol{r}}_{\imath,}^{+}, \\
& g_{1}(r)=\frac{\mathrm{e}^{i k_{1} r}}{r}\left(k_{0}^{2}+\begin{array}{c}
1 k_{0} \\
r
\end{array}-\begin{array}{r}
1 \\
r^{2}
\end{array}\right) \text {, } \\
& g_{2}(r)=\mathrm{e}^{1 k_{11} r}\left(-k_{0}^{2}-\begin{array}{c}
31 k_{0} r \\
r
\end{array}+\begin{array}{l}
3 \\
r^{2}
\end{array}\right)
\end{aligned}
$$

Interaction through reflected contributions follows from the integral in (10), where the important factor is $\mathbf{T}^{-}(k)$, given as

$$
\begin{aligned}
& T_{i t t}^{-}=\left[\left(k^{2}-k_{01}^{2}\right) R_{e}(k)-k_{i)}^{2} R_{m}(k)\right] h\left(t_{l j}, s_{l y}\right)+k k_{0}^{2} R_{m}(k) J_{0}\left(k s_{l j}\right), \\
& T_{11 \geq 2}^{-}=R_{e}(k) k^{3} J_{0}\left(k s_{\eta}\right) \text {, }
\end{aligned}
$$

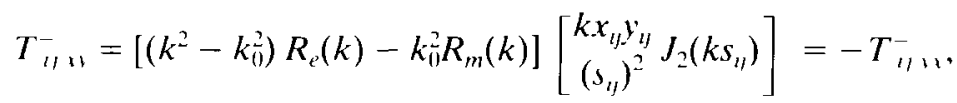

$$
\begin{aligned}
& T_{\| z}^{-}=\left[R_{t}(k)\left(\begin{array}{c}
\left(k^{2}-k_{1)}^{2}\right)^{1 / 2} k^{2} t_{l l} \\
s_{l l}
\end{array}\right) J_{1}\left(k s_{l \prime}\right)\right]=-T_{1 / z r}^{-}
\end{aligned}
$$

In eqs (12) $J_{0}, J_{1} J_{2}$ represent the well-known Bessel functions of the first kınd Further the following definitions were used 


$$
\begin{aligned}
& h(t, s)=J_{1}(k, s) / s-k t^{2} J_{2}(k s) / s^{2}, \quad s=\left(r^{2}+r^{2}\right)^{1 / 2}, \\
& R_{i}(k)=\begin{array}{l}
\tilde{\varepsilon}\left(k^{2}-k_{0}^{2}\right)^{1 / 2}-\left(k^{2}-\tilde{\varepsilon} k_{0}^{2}\right)^{1 / 2} \\
\tilde{\varepsilon}\left(k^{2}-k_{0}^{2}\right)^{1 / 2}+\left(k^{2}-\tilde{\varepsilon} k_{0}^{2}\right)^{1 / 2},
\end{array} \\
& R_{m}(k)=\begin{array}{l}
\left(k^{2}-k_{10}^{2}\right)^{1 / 2}-\left(k^{2}-\bar{\varepsilon} k_{i 1}^{2}\right)^{1 / 2} \\
\left(k^{2}-k_{11}^{2}\right)^{1 / 2}+\left(k^{2}-\tilde{\varepsilon} k_{11}^{2}\right)^{1 / 2}
\end{array}
\end{aligned}
$$

$t$ represents both $x$ and $y$ Since $x, y$ are un itected under reflection the \pm upper index can be neglected in $x, y$ and $s$ represents again the complex relative dielectric constant of the dielectric alt-space The dyadics for the interaction matrix are defined now as

$$
\begin{aligned}
& \mathbf{A}_{u}^{\prime \prime}=\mathbf{C}_{1}-\int_{0}^{r} \mathrm{~d} k \mathrm{e}_{u_{0}}^{\left.-u_{u}\right)\left(z_{u}\right)} a^{3} \mathbf{T}_{n l}^{-}(k) .
\end{aligned}
$$

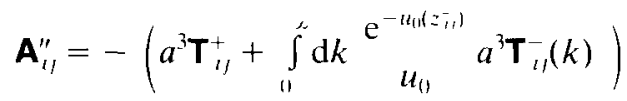

Each of the components of (14) can be screr ied in exactly the same manner as in (8), which is probably the only way to btain realistic predictions from this model As a result after solving of the ir sraction equations the separate dipole values $\boldsymbol{p}$ or $\boldsymbol{\pi}$ are obtaned This is al what is required for further use later on

\section{Remote fields: intensity pattern}

In general what is wanted in explainıng I tyleigh scatterıng experıments, is the intensity at large distances from the luminated spot at the surface The remote electric fields follow in two step First define the total polarizability $\boldsymbol{\alpha}_{\mathrm{tot}}$ for the surface molecule

$\boldsymbol{\alpha}_{\mathrm{tot}}=4 \pi \alpha_{0} \sum_{i j=1}^{N} \mathbf{A}_{i j}^{-1}$

Defining next the Fresnel reflection matrix $1(\theta)$ as

$$
\begin{aligned}
& \mathbf{R}(\theta)=\left\|\begin{array}{lll}
1+R_{m}(\theta) & 0 & 0 \\
0 & 1+R_{\mathrm{e}}(\theta) & 0 \\
0 & 0 & 1+R_{m}(t
\end{array}\right\| \\
& R_{e}(\theta)=\begin{array}{l}
\bar{\varepsilon} \cos \theta-\left(\bar{\varepsilon}-\sin ^{2} \theta\right)^{1 / 2} \\
\bar{\varepsilon} \cos \theta+\left(\bar{\varepsilon}-\sin ^{2} \theta\right)^{1 / 2}
\end{array} \quad R_{m}(\theta)=\begin{array}{l}
\operatorname{si} \theta-\left(\bar{\varepsilon}-\sin ^{2} \theta\right)^{1 / 2} \\
\mu \theta+\left(\bar{\varepsilon}-\sin ^{2} \theta\right)^{1 / 2}
\end{array}
\end{aligned}
$$

Here, as in ref [13], the presupposition $w$, used that the $x$-axis coincides 
with the projection of the incident wavevector The angle of this wavevector with the $x$-axis is $\theta_{0}$. Now the total polarizability of the molecule $p_{\text {tot }}$ is connected to the incident field $\boldsymbol{E}_{\text {inc }}$ through

$\boldsymbol{p}_{\mathrm{tot}}=\boldsymbol{\alpha}_{\mathrm{tot}} \mathbf{R}\left(\theta_{0}\right) \boldsymbol{E}_{\mathrm{inc}}^{\prime}$

Since we suppose also that the size of the molecule is much smaller than the wavelength of the light used, the following treatment of the second step is allowed Using the asymptotic solutions given in ref [15], the remote field $\boldsymbol{E}_{\mathrm{rcm}}$ detected at $\boldsymbol{R}$, where the corresponding unit vector makes an angle $\theta$ with the $z$-axis, follows from

$\boldsymbol{E}_{\mathrm{r} \mathrm{m}}(\boldsymbol{R})=k_{01}^{2} \frac{\mathrm{e}^{\mathrm{k} k_{\mathrm{l}} R}}{4 \pi \varepsilon_{0} R} R \boldsymbol{\tau}_{\mathrm{rem}} \mathbf{R}^{\prime}(\theta) \boldsymbol{\alpha}_{\mathrm{tot}} \mathbf{R}\left(\theta_{0}\right) \boldsymbol{E}_{\mathrm{ln \iota}}$,

where we used $R=|\boldsymbol{R}|$ and the following definitıons

$\mathbf{\tau}_{\mathrm{rcm}}=\mathbf{1}-\hat{\boldsymbol{R}} \hat{\boldsymbol{R}}$,

$\mathbf{R}^{\prime}(\theta)=\left\|\begin{array}{lll}1+R_{m}(\theta) & 0 & 0 \\ 0 & 1+R_{m}(\theta) & 0 \\ 0 & 0 & 1+R_{e}(\theta)\end{array}\right\|$

To calculate next the intensities is the standard procedure

$I_{\mathrm{rem}}(\boldsymbol{R})=\frac{1}{2} \boldsymbol{E}_{\mathrm{rem}}^{*}(\boldsymbol{R}) \cdot \boldsymbol{E}_{\mathrm{rum}}(\boldsymbol{R})$

The expression is valid of course except for the specular beam direction

\section{Discussion}

Expressions (19) and (20) predict the Rayleigh scattered intensity produced by a large molecule on top of a dielectric surface, as can be measured To our opinion the initial stages of the formation of polysylanes made by means of HOMOCVD on top of a cleavage plane of crystalline silicon, come close to such a description As such this theory can be seen, after incorporation of the proper statistics, as a first-order approximation of the scattered intensity for that case Predicted and measured intensities should match in an absolute way Especially the polarization-dependent observations yield useful information Taking into account however the existing experience about light-scattering from gaseous alkanes, it is sure that for adequate matching of theoretical and experimental intensities or depolarization ratios $\varepsilon$, has to be tuned around 10 and depending upon the type of adsorption $\varepsilon_{\mathrm{r}}$ should be given a value of 1 (physisorption) or higher (chemisorption) If matching results, it learns us that the optical behaviour of covalently bonded 
materials, especially silicon is properly de: ribed by means of a screened induced-dipole model This would pave the way for reliable calculations of the optical response of more complicated si zon-based configurations

\section{References}

[1] W E Spedr and P G Le Comber. Solid State C( Imun 17 (1975) 1193

[2] G Lucovsky, R J Nemanich and J C Knights, I ys Rev B19 (1973) 2064

[3] B A Scott, J A Reımer, R M Plecenık, E E Sın nyı and W Reuter, Appl Phys Letters 40 (1982) 973

[4] D J Woltord, B A Scott J A Reımer and J A Beradlev, Physica 117/118B (1983) 920

[5] S Furukawd and N Matsumoto, Soldd State Cot nun 48 (1983) 539

[6] D J Woltord. J A Reimer and B A Scott Apr Phvs Letters 42 (1983) 369)

[7] W E Pickett. Phys Rev B23 (1981) 6603

[8] D A Papaconstantopoulos and E N Economou 'hys Rev B24 (1981) 7233

[9] K Takeda N Matsumoto and M Fukuchı, Phy Rev B30 (1984) 5871

[10] B M Ladanyı and T Keyes, in Advances in Ch. ucal Physics Vol 16, Eds I Prigogine and S A Rice (Wiley-Interscience, New York : 9), and reterences thereın

[11] C Wijers, to be published

[12] T E Furtak and J Reyes Surface Scr 93 (198()) 51

[13] S Efrima and H Metiu, J Chem Phys $70(197$ 1602

[14] S Efrima and H Metiu J Chem Phys 70)(197 2297

[15] J R Watt, Electromagnetic Wave Theory (Harp and Rou, New Yorh, 1984) 